\title{
Influence of enhanced recovery after surgery programs on laparoscopy-assisted gastrectomy for gastric cancer: a systematic review and meta-analysis of randomized control trials
}

\author{
Zhengyan Li, Qian Wang, Bofei Li, Bin Bai and Qingchuan Zhao*
}

\begin{abstract}
Background: This meta-analysis is aimed to evaluate the feasibility and safety of enhanced recovery after surgery (ERAS) programs in gastric cancer patients undergoing laparoscopy-assisted gastrectomy (LAG).

Methods: We performed a meta-analysis of randomized control trials involving either enhanced recovery after surgery (ERAS)/fast track surgery (FTS) for patients underwent LAG. EMBASE, Pubmed, Web of science, and Cochrane Library were searched. Primary outcomes included the length of postoperative hospital stay, cost of hospitalization, postoperative complications, and readmission rate.

Results: Five randomized control trials were eligible for analysis. There were 159 cases in FTS group and 156 cases in conventional care group. Compared with conventional care group, FTS group relates to shorter postoperative hospital stay (WMD $-2.16 ; 95 \% \mathrm{Cl}-3.05$ to $-1.26, P<0.00001$ ), less cost of hospitalization (WMD $-4.72 ; 95 \% \mathrm{Cl}-6.88$ to $-2.55, P$ $<0.00001$ ), shorter time to first flatus (WMD $-9.72 ; 95 \% \mathrm{Cl}-13.75$ to $-5.81, P<0.00001)$, lower level of C-reaction protein on postoperative days 3 or 4 (WMD -19.66 ; $95 \% \mathrm{Cl}-28.98$ to $-10.34, P<0.00001$ ), higher level of albumin on postoperative day 4 (WMD 3.45; 95\% Cl 2.01 to 4.89, $P<0.00001$ ), and postoperative day 7 (WMD 5.63; $95 \% \mathrm{Cl} 1.01$ to $10.24, P=0.02$ ). Regarding postoperative complications, no significant differences were observed between FTS group and conventional care group (OR $0.63,95 \% \mathrm{Cl} 0.37$ to $1.09, P=0.10$ ). The readmission rate of FTS group was comparable to conventional care group (WMD 3.14; 95\% Cl 0.12 to 81.35, $P=0.49$ ).
\end{abstract}

Conclusions: Among patients undergoing LAG, FTS is associated with shorter postoperative hospital stay, rapid postoperative recovery, and decreased cost without increasing complications or readmission rate. The combined effects of the two methods could further accelerate clinical recovery of gastric cancer patients.

Keywords: Fast track surgery, Enhanced recovery after surgery, Gastric cancer, Laparoscopy-assisted gastrectomy

\footnotetext{
* Correspondence: zhaoqc@fmmu.edu.cn

Department of Surgery, Xijing Hospital of Digestive Diseases, The Fourth

Military Medical University, No. 127 Changle West Road, Xian 710032, China
} 


\section{Background}

Gastric cancer is a worldwide health concern and is the second leading cause of cancer-related deaths in China [1]. In recent years, the use of laparoscopy-assisted gastrectomy (LAG) was developed and clinically implemented to treat gastric cancer with the advantage of better short-term outcomes [2-4]. Fast track surgery (FTS)/enhanced recovery after surgery (ERAS) was first introduced by Kehlet in the 1990s and has gained satisfactory curative effect in many fields of surgery [5-10]. ERAS guidelines have been established in many kinds of surgeries, such as colectomy, cystectomy, and stomach surgery. Recent meta-analyses have demonstrated that ERAS is safe and effective after laparoscopic hepatectomy and colorectal surgery [11-13]. Previous studies have demonstrated that ERAS could accelerate the postoperative recovery in open gastrectomy for gastric cancer [14-16]. However, the role of ERAS in LAG is still unclear. To date, several studies have reported the value of FTS in LAG [17-19]. But they are all based on singlecenter studies with small sample size which may influence the credibility of the results. Therefore, we conduct this meta-analysis to assess the effects of ERAS protocol in gastric cancer patients undergoing LAG.

\section{Methods}

\section{Literature search}

This meta-analysis was conducted on the basis of the preferred reporting items for systematic reviews and meta-analyses (PRISMA) guidelines [20]. EMBASE, Pubmed, Web of science, and Cochrane Library were searched from January 1995 to July 2017. Studies were limited to English and Chinese. We used the following key words: "fast track," "enhanced recovery," "FTS," "ERAS," "gastric cancer," "laparoscopy-assisted gastrectomy," and "laparoscopic gastrectomy." Additionally, the reference lists of all included studies were also searched to retrieve related articles.

\section{Inclusion and exclusion criteria}

Inclusion criteria categories included (1) patients undergoing LAG for gastric cancer (2) perioperative care using either ERAS or FTS protocols compared with standard or conventional care, (3) randomized controlled trials (RCTs)(4) clearly state the ERAS program, and at least one or more of the primary outcomes was reported. Studies were excluded if they (1) included less than 6 interventions items in the FTS group according to the ERAS guidelines [21] (2) unable to provide one of the primary outcome mentioned above.

\section{Outcome measures}

The primary outcomes were the length of postoperative hospital stay, cost of hospitalization, postoperative complications, and readmission rate. The secondary outcomes were time to first flatus, level of C-reaction protein, albumin, and Interleukin-6.

\section{Quality assessment and data extraction}

The quality of each included RCT was assessed according to the Cochrane methodology, which included the following evaluation domains: random sequence generation, allocation concealment, blinding of participants and personnel, blinding of outcome assessment, incomplete outcome data, selective reporting, and other biases [22]. The data was extracted from each eligible trial by two authors (Q Wang and Bin Bai). From each study, we extracted the general information of included studies, including the author, publication year, type of studies, sample size, surgery method, follow-up duration, and some other details.

\section{Statistical analysis}

The data pooling was carried out using the Review Manager software (version 5.3, Nordic Cochrane Centre). The pooled results were expressed as the mean difference (MD) with 95\% confidence interval (CI). Continuous variables were assessed using weighted mean difference (WMD). Dichotomous variables was analyzed using odds ratios (OR) and 95\% CI.

Statistical heterogeneity among studies was evaluated by using the Cochran $Q$ statistic and quantified by $I^{2}$ statistics. The random-effects model was used in this meta-analysis. Due to the limited number of studies (less than 10), the funnel plot was not performed to test the publication bias. $P<0.05$ was considered statistically significant.

\section{Result}

\section{Characteristics of trials}

Figure 1 summarizes the flow chart for the selection of eligible studies. Eventually, five studies [17-19, 23, 24] were considered eligible for this meta-analysis. In all, data from 315 patients were recorded, of which 159 in the FTS group and 156 in the conventional care group. All included studies were conducted in Asia (4 in China and 1 in Korea), and their studies were published between 2012 and 2016. Table 1 shows the general characteristics of included studies. The EARS items applied in the included studies are presented in Table 2. Regarding the methodological quality, all included studies showed low to moderate overall risks of bias (Fig. 2).

\section{Meta-analysis results}

Postoperative hospital stay and hospitalization expenditure The data of postoperative hospital stay could be obtained from four included studies. The result showed 


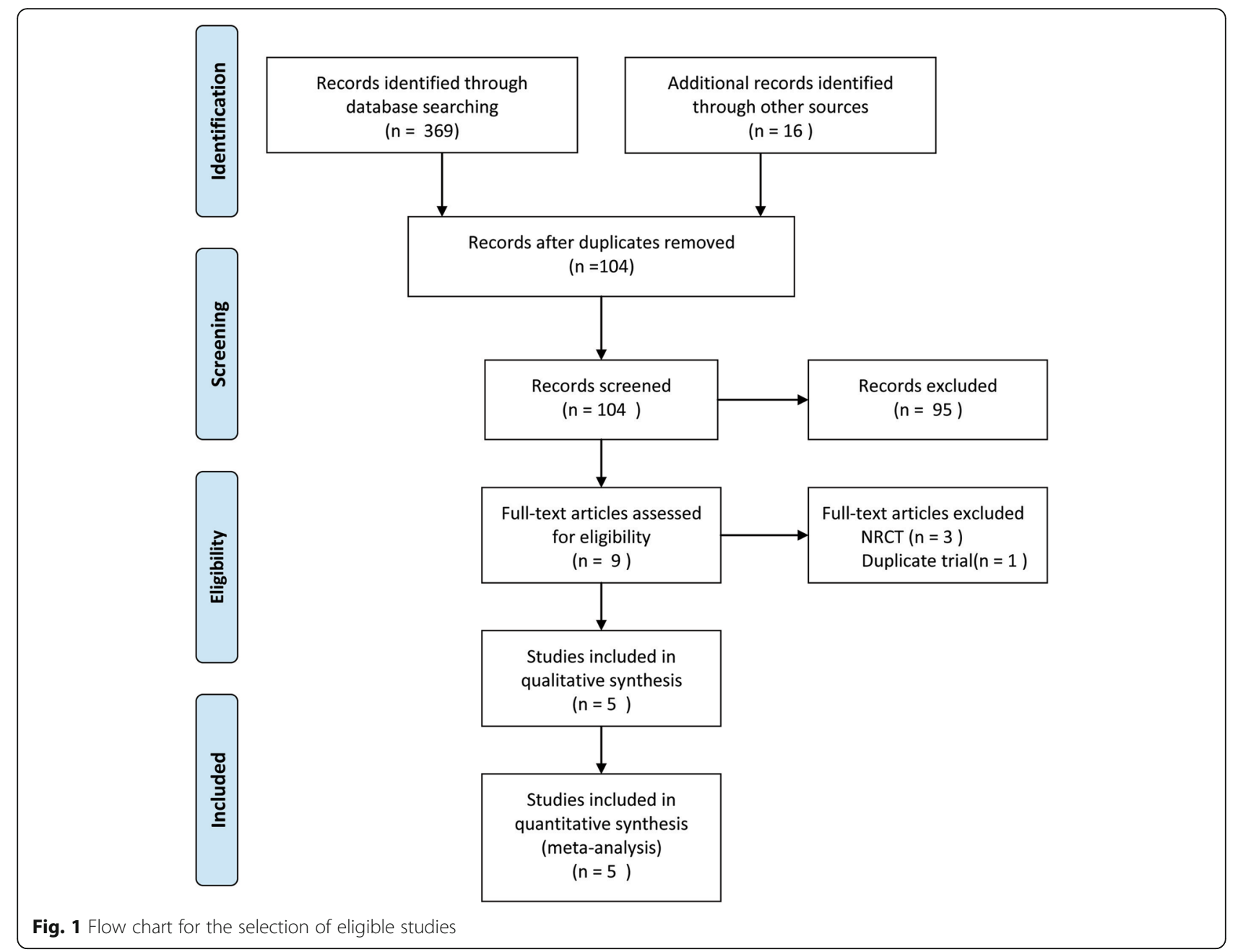

that FTS group is associated with a significant reduction in postoperative hospital stay when compared to the conventional care group (WMD - 2.16; 95\% CI 3.05 to $-1.26, P<0.00001$ ) (Fig. 3a). High heterogeneity was observed among the studies $\left(P=0.04, I^{2}=\right.$ $65 \%$ ), and a random-effects model was used. Three included studies reported the cost of hospitalization. Results showed that FTS group had a less cost of hospitalization compared to the conventional care group (WMD - 4.72; $95 \% \mathrm{CI}-6.88$ to $-2.55, \quad P<$ 0.00001) (Fig. 3b).

\section{Postoperative complications and readmission rate}

Postoperative complications were described in all five studies. The results of this meta-analysis did not show a significant difference between the two groups $(\mathrm{OR}=$ $0.63,95 \%$ CI 0.37 to $1.09, P=0.10$ ) (Fig. 3c). Two studies reported readmission rate of patients. No statistical

Table 1 Characteristics of included studies

\begin{tabular}{|c|c|c|c|c|c|c|c|c|c|c|c|}
\hline \multirow[t]{2}{*}{ Reference } & \multirow[t]{2}{*}{ Year } & \multirow{2}{*}{$\begin{array}{l}\text { Type } \\
\text { of } \\
\text { study }\end{array}$} & \multicolumn{2}{|c|}{ Sample size } & \multirow{2}{*}{$\begin{array}{l}\text { Surgery } \\
\text { method }\end{array}$} & \multirow[t]{2}{*}{ Outcomes } & \multirow{2}{*}{$\begin{array}{l}\text { Follow-up } \\
\text { duration }\end{array}$} & \multicolumn{2}{|l|}{ Age } & \multicolumn{2}{|l|}{ BMl } \\
\hline & & & FTS & CC & & & & FTS & CC & FTS & CC \\
\hline Kim et al. & 2012 & $\mathrm{RCT}$ & 22 & 22 & LAG & 1.2.4.5.6.7 & 2 weeks & $52.64 \pm 11.5$ & $57.45 \pm 14.54$ & $23.40 \pm 3.17$ & $23.77 \pm 3.54$ \\
\hline Hu et al. & 2012 & $\mathrm{RCT}$ & 19 & 22 & LAG & 1.2.4.5.6.7 & 4 weeks & $59(49-71)$ & $62.5(45-72)$ & $22.94 \pm 2.23$ & $22.99 \pm 2.24$ \\
\hline Abdikarim et al. & 2015 & $\mathrm{RCT}$ & 30 & 31 & LAG & 1.4 .7 & 30 days & $63 \pm 12$ & $62 \pm 11$ & $N R$ & NR \\
\hline Li et al. & 2016 & $\mathrm{RCT}$ & 67 & 60 & LAG & 1.4.7.8 & 2-21 months & $72.9 \pm 6.7$ & $71.8 \pm 8.0$ & $21.1 \pm 2.5$ & $20.4 \pm 2.3$ \\
\hline Liu et al. & 2016 & $\mathrm{RCT}$ & 21 & 21 & LAG & 1.2.3.4.5.7.8 & NR & $69.2 \pm 5.1$ & $70.3 \pm 5.8$ & $21.5 \pm 2.0$ & $21.9 \pm 2.3$ \\
\hline
\end{tabular}

$R C T$ randomized controlled trials, FTS fast track surgery, CC conventional care, NR not reported, 1 time to first flatus, 2 C-reaction protein, 3 interleukin-6, 4 length of postoperative hospital stay, 5 hospitalization expenditure, 6 readmission rate, 7 postoperative complications, 8 albumin 
Table 2 EARS/FTS elements applied in the included studies

\begin{tabular}{|c|c|c|c|c|c|}
\hline Element & Kim et al. & Hu et al. & Abdikarim et al. & Li et al. & Liu et al. \\
\hline Preoperative counseling & $\sqrt{ }$ & $\sqrt{ }$ & $\sqrt{ }$ & $\sqrt{ }$ & $\sqrt{ }$ \\
\hline Avoid preoperative bowel preparation & $\sqrt{ }$ & $\sqrt{ }$ & $\sqrt{ }$ & $\sqrt{ }$ & $\sqrt{ }$ \\
\hline Preoperative carbohydrate loading & $\sqrt{ }$ & $\sqrt{ }$ & $\sqrt{ }$ & $\sqrt{ }$ & $\sqrt{ }$ \\
\hline No pre-anesthetic medication & $\sqrt{ }$ & $\sqrt{ }$ & $\sqrt{ }$ & $\sqrt{ }$ & $\sqrt{ }$ \\
\hline \multicolumn{6}{|l|}{ Prophylaxis against thromboembolism } \\
\hline \multicolumn{6}{|l|}{ Antimicrobial prophylaxis } \\
\hline Standard anesthetic & & & $\sqrt{ }$ & & \\
\hline \multicolumn{6}{|c|}{ Postoperative nausea and vomiting prophylaxis } \\
\hline Minimal invasive surgery & $\sqrt{ }$ & $\sqrt{ }$ & $\sqrt{ }$ & $\sqrt{ }$ & $\sqrt{ }$ \\
\hline Avoid nasogastric tube & $\sqrt{ }$ & $\sqrt{ }$ & $\sqrt{ }$ & $\sqrt{ }$ & $\sqrt{ }$ \\
\hline Prevent hypothermia & $\sqrt{ }$ & & & $\sqrt{ }$ & \\
\hline Perioperative fluid management & & & $\sqrt{ }$ & & $\sqrt{ }$ \\
\hline Avoid peritoneal drainage & & $\sqrt{ }$ & & & \\
\hline Early urinary removal & $\sqrt{ }$ & $\sqrt{ }$ & $\sqrt{ }$ & $\sqrt{ }$ & $\sqrt{ }$ \\
\hline Postoperative analgesia & $\sqrt{ }$ & & $\sqrt{ }$ & $\sqrt{ }$ & $\sqrt{ }$ \\
\hline Early oral feeding & $\sqrt{ }$ & $\sqrt{ }$ & $\sqrt{ }$ & $\sqrt{ }$ & $\sqrt{ }$ \\
\hline Early mobilization & $\sqrt{ }$ & $\sqrt{ }$ & $\sqrt{ }$ & $\sqrt{ }$ & $\sqrt{ }$ \\
\hline Audit & & & & & \\
\hline
\end{tabular}

difference was found between the two groups (WMD = 3.14; $95 \%$ CI 0.12 to 81.35 ; $P=0.49$ ) (Fig. $3 d$ ).

Time to first flatus, ambulation time, and time to start diet The results of this meta-analysis revealed that FTS group was associated with a shorter time to first flatus (WMD - 9.78; 95\% CI -13.75 to $-5.81, P<0.00001$ ) (Fig. 4a). No significant differences were found between the two groups in terms of ambulation time (WMD 0.97 ; $95 \% \mathrm{CI}-2.27$ to $0.33, P=0.14$ ) (Fig. $4 \mathrm{~b}$ ) and time to start diet (WMD $-1.30 ; 95 \% \mathrm{CI}-2.87$ to $0.26, P=$ 0.10) (Fig. 4c).

\section{C-reaction protein, albumin, and Interleukin-6}

Three studies reported the C-reaction protein level on postoperative day 1 . There was no significant difference between the two groups (WMD $=-8.34 ; 95 \% \mathrm{CI}$ -25.05 to $8.37, P=0.33$ ) (Fig. $4 d$ ). Results suggested that FTS group was associated with a lower level of C-reaction protein on postoperative days (PODs) 3 and 4 (WMD - 19.66; 95\% CI -28.98 to $-10.34, P<$ $0.00001)$, and there was no significant difference between the two groups on postoperative day 7 (WMD - 18.65; 95\% CI -38.75 to $1.46, P=0.07$ ) (Fig. $4 \mathrm{~d}$ ). Two studies reported the albumin level on postoperative day 1 . No significant difference was observed between the two groups (WMD $=0.36$; $95 \%$ CI -0.56 to 1.27, $P=0.45$ ) (Fig. 4e). However, the results showed that FTS group was associated with a higher level of albumin on postoperative day 4 (WMD 3.45 ; $95 \%$ CI
2.01 to $4.89, P<0.00001)$ and postoperative day 7 (WMD 5.63; 95\% CI 1.01 to $10.24, P=0.02$ ) (Fig. 4e).

\section{Discussion}

ERAS was first proposed by the Danish physician Kehlet, with the aim of reducing surgical trauma and facilitating postoperative recovery by the use of a series of perioperative management [5]. Nowadays, laparoscopic surgery have garnered tremendous popularity over open surgery with better short-term outcomes, such as less operative bleeding, earlier bowel movement, reduced pain, fewer overall complications, and shorter postoperative hospital stays [25-27]. To date, ERAS and laparoscopic technique have been widely applied. However, the benefit of ERAS in patients undergoing laparoscopic gastrectomy is still unclear. To the best of our knowledge, this is the first meta-analysis focus on this topic. Comparing with previous meta-analyses mainly focus on open gastrectomy, the surgical procedure of our study was limited to LAG.

The results of the meta-analysis suggest that the FTS group is associated with a significant reduction in postoperative hospital stay, time to first flatus, postoperative CRP, IL-6, and hospital charge as compared with conventional care group. Additionally, no difference in postoperative complications and readmission rate was observed when comparing ERAS and conventional care within LAG. 


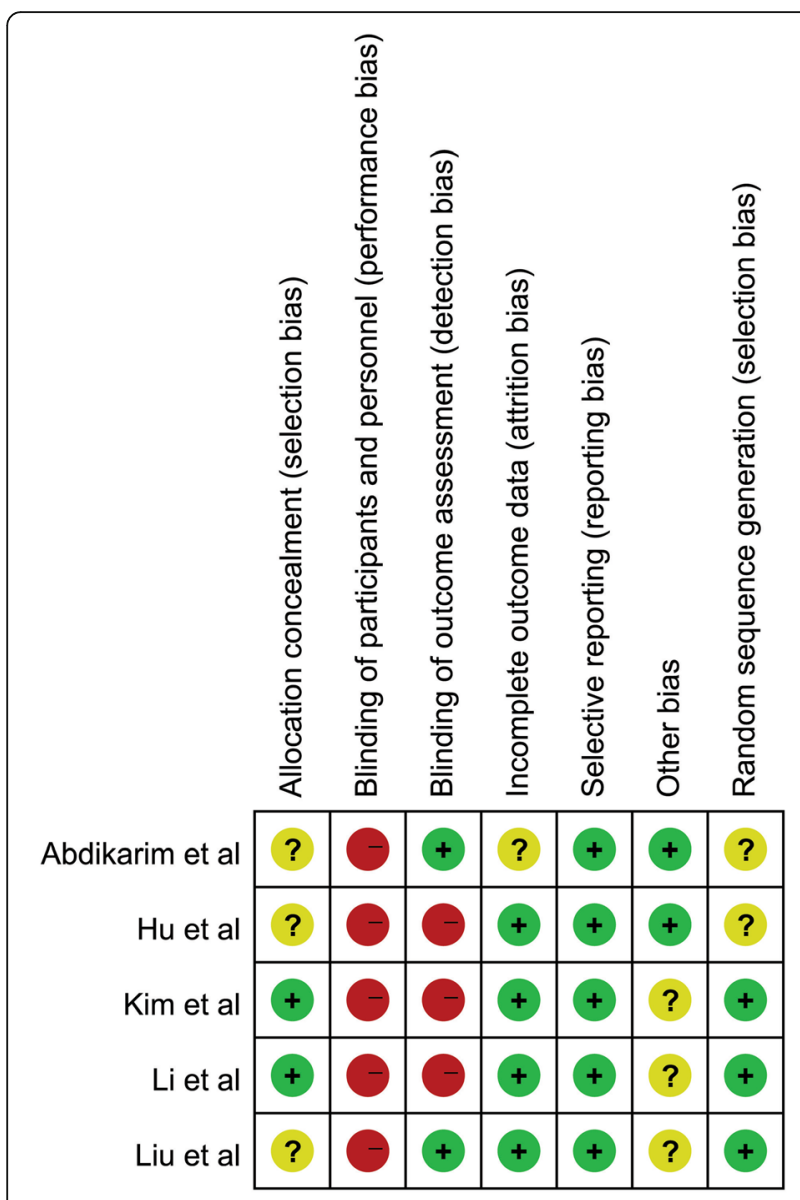

Fig. 2 Risk of bias summary of all included studies. Plus low risk of bias, minus high risk of bias, question mark unclear risk of bias

A shorter hospital stay was the advantage of laparoscopic surgery. Our results showed that ERAS combined with LAG could shorten the length of postoperative hospital stay as compared with conventional care. Meanwhile, we also found that all included studies showed a consistent tendency favoring the FTS group. A previous meta-analysis suggested that ERAS combined with LAG are associated with a significant reduction in postoperative hospital stay of 2.68 days as compared with conventional care. Our meta-analysis showed that FTS group was associated with a reduction in postoperative hospital stay of 2.16 days. Secondly, we found in the present study that FTS group are associated with a significant reduction in time to first flatus. Four included studies with appropriate data that reported this outcome showed a result favoring FTS group. It has been widely accepted that both LAG and ERAS can reduce surgical trauma and facilitating postoperative recovery. Our results revealed that the combined effects of the two methods can further accelerate clinical recovery of the patients undergoing LAG.
Postoperative complication is the key indicator for assessing the safety and feasibility of surgical procedure. Extensive studies have shown that LAG is associated with fewer complications, such as incision infection and pneumonia as compared with OG. Meanwhile, one of the main principles of the ERAS protocol is reducing postoperative complications. Previous meta-analyses and RCTs showed reduced postoperative complications and readmission rate when ERAS was implemented in OG $[14,16,28-30]$. A previous study in our department demonstrated that the postoperative complication rate in the FTS group was lower than that in the conventional care group. They found that the ERAS protocol could reduce the incidence of pneumonia. This benefit may mainly attribute to the early ambulation of patients [30]. Li et al. [24] also reported that the FTS group was associated with a reduction in postoperative complication rate following LAG. Our result also showed a similar tendency favoring FTS, but there was no significant difference between the two groups. The statistical insignificance may be attributed to the reduction of some common complications such as incision infection, and pneumonia may have already been achieved by LAG, leaving little room for improvement via the implement of ERAS protocol. Additionally, it may also due to the relative small sample size of the present study.

The use of abdominal drains following gastrectomy still remains controversial. Prophylactic peritoneal drainage has been widely used during gastrointestinal surgery because of several advantages such as removing intraperitoneal fluid and assisting the early detection of postoperative hemorrhage or anastomotic leakage [31-33]. However, peritoneal drainage can cause uncomfortable which may limit early mobilization and postoperative recovery. There is evidence that abdominal drains do not reduce the complication rate but increase intraperitoneal fluid collection, infections, and risk of occurrence of postoperative fistula [34]. Despite of these disadvantages, peritoneal drainage is still commonly used for gastric cancer surgery at most institutions. Only one of the included studies in this meta-analysis did not use peritoneal drainage as routine treatment [18]. Thus, the safety and efficacy of no routine use of abdominal drainage tube need to be assessed in further investigation.

It have been recognized widely that the advantages of laparoscopic surgery such as alleviating surgical stress and reducing respiratory interference could accelerate postoperative recovery [35-37]. To date, some authors have reported that ERAS protocol could accelerate recovery by mitigating the inflammatory response $[14,15,38,39]$. Our results suggest the combination of the two methods can further alleviate the inflammation and immune inhibition based on the efficacy of a single method. Liu et al. [40] indicated 

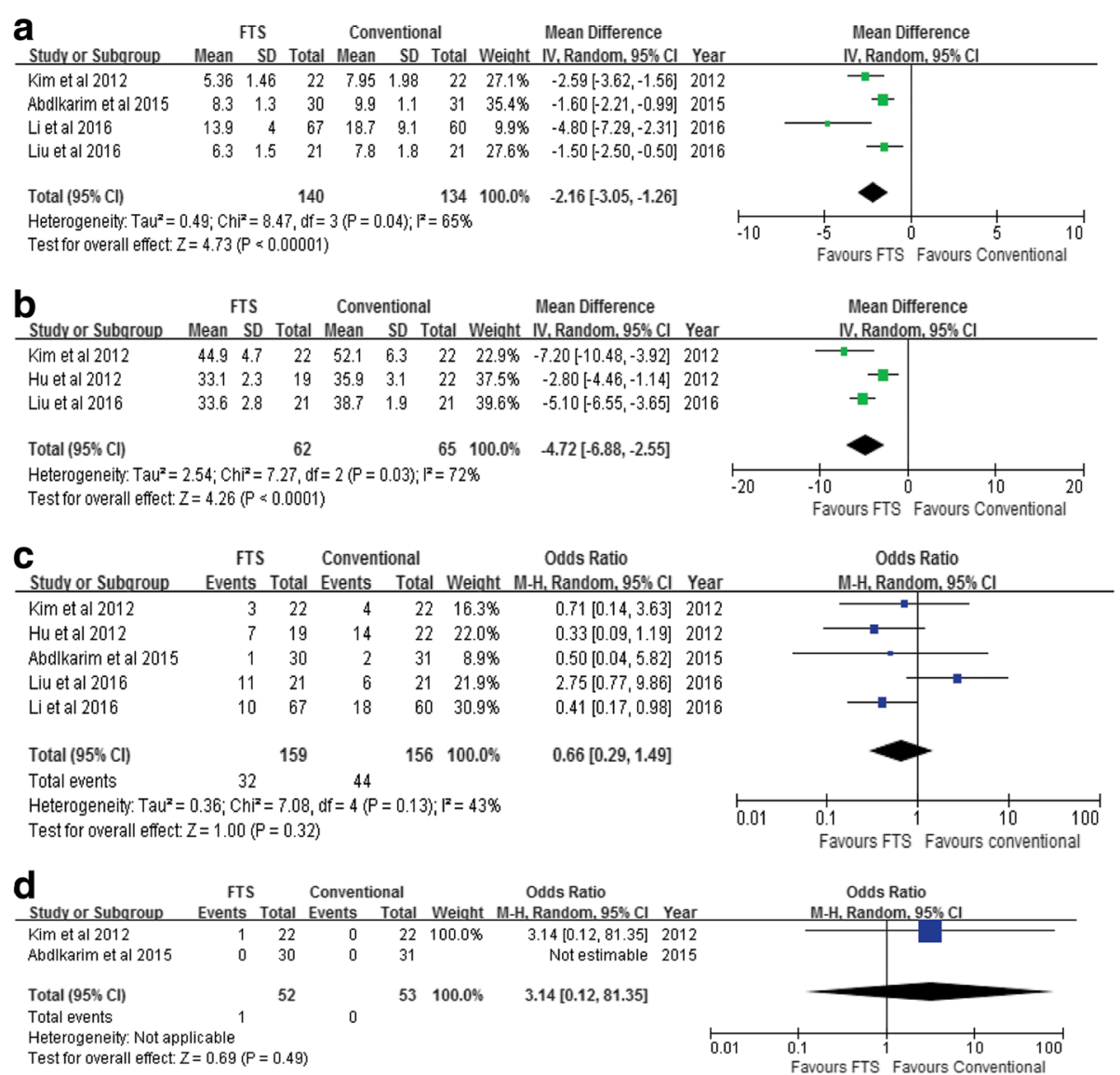

Fig. 3 Meta-analyses of primary outcomes. a Postoperative hospital stay. b Cost of hospitalization. c Postoperative complications. d Readmission rate

that FTS group showed lower C-reaction protein and IL-6 levels (postoperative days 1, 4, and 7) compared with the conventional care group. The level of serum albumin is a nutritional status indicator. Our results showed that the albumin in the FTS group was higher than that in the conventional perioperative care group on postoperative days 4 and 7 . We presumed that this difference may mainly attributed to the early enteral nutrition (EN) improved postoperative nutrition status in FTS group. In addition, several meta-analyses have demonstrated that early EN may decrease early occurrence of postoperative infections, shorten the length of hospital stay and therefore promote the postoperative recovery of paitents [41-45].

Researches have shown that laparoscopic surgery is associated with better quality of life (QOL) as compared with open surgery. Regarding the QOL following ERAS combined with LAG, studies have been seldom reported. Kim et al. [17] reported that the ERAS protocol may have no negative effect on QOL or patient satisfaction. Moreover, certain aspects of QOL such as pain, fatigue, appetite loss, and financial problems can be improved by the implement of ERAS protocol. Our study has several limitations. First, the included studies are all from Aisa and in relatively small sample size, which may limited the generalization of our results. Second, the differences in patient characteristics is a source of bias that may affect the stability of the results. Third, although time to first flatus had low heterogeneity, other outcomes had moderate or high heterogeneity. Moreover, the compliance is varied among all included studies which may reduce the benefits of ERAS protocol.

\section{Conclusions}

In conclusion, this meta-analysis indicates that ERAS combined with laparoscopic technique is safe and effective for gastric cancer and could significantly decrease postoperative hospital stay, cost of hospitalization, and time to first flatus without increasing postoperative complication and readmission rate. High-quality and larger-scale studies are needed to provide more solid evidence. 


\begin{tabular}{|c|c|c|c|c|c|c|c|c|c|}
\hline a & & FTS & & Con & ientiona & & & Mean Difference & \\
\hline Study or Subaroup & Mean & SD & Total & Mean & SD & Total & Weight & IV. Random, $95 \% \mathrm{Cl}$ & \\
\hline Hu et al 2012 & 55.75 & 9.25 & 19 & 65 & 7.75 & 22 & $56.7 \%$ & $-9.25[-14.52,-3.98]$ & 20 \\
\hline Kim et al 2012 & 63.05 & 18.62 & 22 & 67.41 & 15.28 & 22 & $15.6 \%$ & $-4.36[-14.43,5.71]$ & \\
\hline Li et al 2016 & 76.8 & 21.6 & 67 & 91.2 & 26.4 & 60 & $22.1 \%$ & $-14.40[-22.85,-5.95]$ & \\
\hline Liu et al 2016 & 48 & 28.8 & 21 & 60 & 26.4 & 21 & $5.6 \%$ & $-12.00[-28.71,4.71]$ & \\
\hline Total $(95 \% \mathrm{Cl})$ & & & 129 & & & 125 & $100.0 \%$ & $-9.78[-13.75,-5.81]$ & \\
\hline
\end{tabular}

b
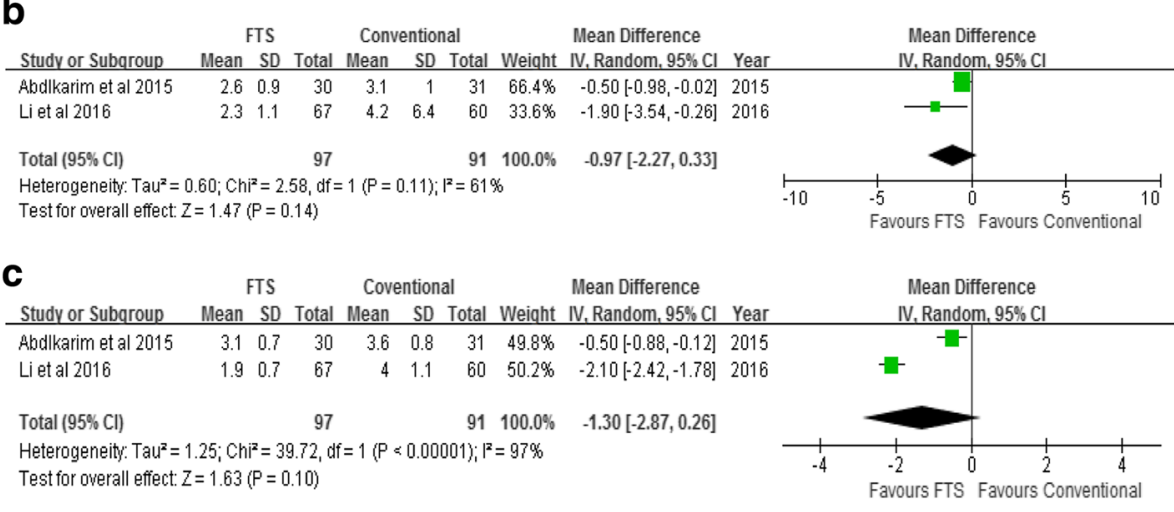

d

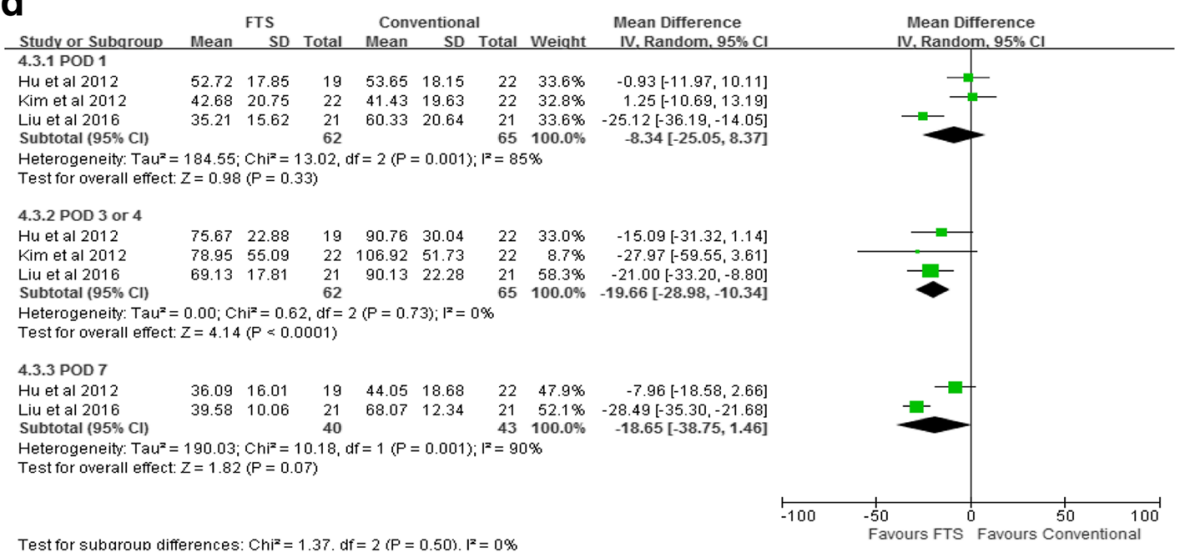

\section{e}

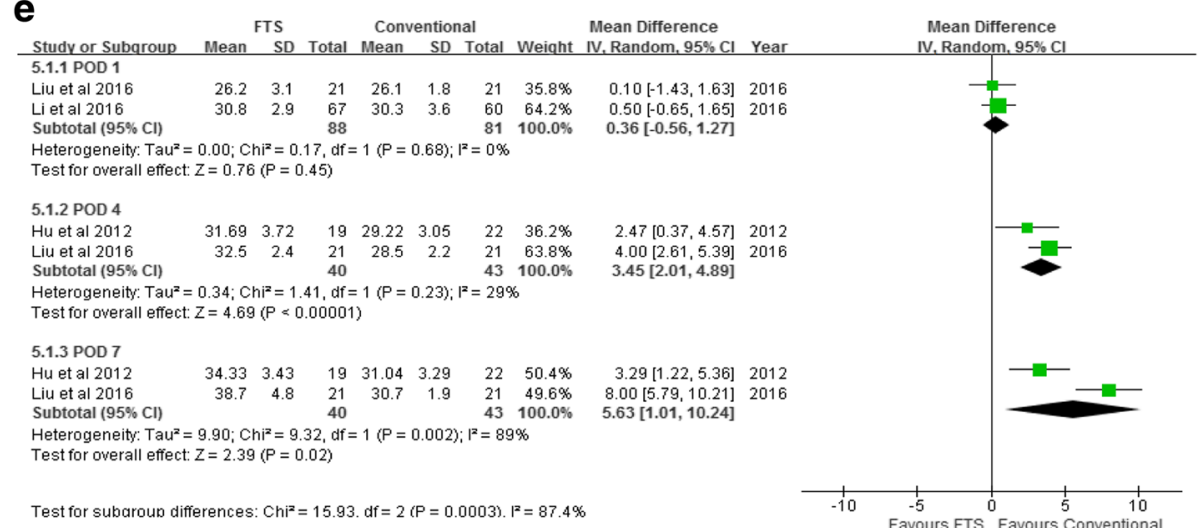

Fig. 4 Meta-analyses of secondary outcomes. a Time to first flatus. b Ambulation time. c Time to start diet. d C-reaction protein. e Albumin 


\section{Acknowledgements}

Not applicable

\section{Funding}

This study was supported by the National Key Basic Research Program of China (no. 2014CBA02002).

\section{Availability of data and materials}

All the data used in the study can be obtained from the original articles.

\section{Authors' contributions}

ZL and QZ performed the experiment conception and design. QW and BB performed the research and retrieved the data. $Z \mathrm{~L}$ and $\mathrm{BL}$ performed the data analysis. ZL did the paper writing. All authors read and approved the final manuscript.

\section{Ethics approval and consent to participate}

All analyses were based on the previous published studies; thus, no ethical approval and patient consent are required.

\section{Consent for publication}

All analyses were based on previous published studies; thus, no consent for publication is required.

\section{Competing interests}

The authors declare that they have no competing interests.

\section{Publisher's Note}

Springer Nature remains neutral with regard to jurisdictional claims in published maps and institutional affiliations.

Received: 13 September 2017 Accepted: 15 November 2017 Published online: 23 November 2017

\section{References}

1. Chen W, Zheng R, Baade PD, Zhang S, Zeng H, Bray F, Jemal A, XQ Y, He J. Cancer statistics in China, 2015. CA Cancer J Clin. 2016;66:115-32.

2. Vinuela EF, Gonen M, Brennan MF, Coit DG, Strong VE. Laparoscopic versus open distal gastrectomy for gastric cancer: a meta-analysis of randomized controlled trials and high-quality nonrandomized studies. Ann Surg. 2012; 255:446-56.

3. Jiang L, Yang KH, Guan QL, Cao N, Chen Y, Zhao P, Chen YL, Yao L. Laparoscopy-assisted gastrectomy versus open gastrectomy for resectable gastric cancer: an update meta-analysis based on randomized controlled trials. Surg Endosc. 2013:27:2466-80

4. Lin JXHC, Zheng CH, Li P, Xie JW, Wang JB, Lu J. Laparoscopy-assisted gastrectomy with D2 lymph node dissection for advanced gastric cance without serosa invasion: a matched cohort study from South China. World J Surg Oncol. 2013;11:4.

5. Bardram L, Funch-Jensen $P$, Jensen $P$, Crawford ME, Kehlet $H$. Recovery after laparoscopic colonic surgery with epidural analgesia, and early oral nutrition and mobilisation. Lancet. 1995;345:763-4.

6. Kehlet H, Wilmore DW. Multimodal strategies to improve surgical outcome. Am J Surg. 2002:83:630-41

7. Grant MC, Yang D, CL W, Makary MA, Wick EC. Impact of enhanced recovery after surgery and fast track surgery pathways on healthcare-associated infections: results from a systematic review and meta-analysis. Ann Surg. 2017;265:68-79.

8. Zhao $Y$, Qin H, Wu Y, Xiang B. Enhanced recovery after surgery program reduces length of hospital stay and complications in liver resection: a PRISMA-compliant systematic review and meta-analysis of randomized controlled trials. Medicine (Baltimore). 2017;96:e7628.

9. Deng $X$, Chen $X$, Huo Z, Shi $Y$, Jin Z, Feng H, Wang Y, Wen C, Qian H, Zhao R, Qiu W, Shen B, Peng C. Modified protocol for enhanced recovery after surgery is beneficial for Chinese cancer patients undergoing pancreaticoduodenectomy. Oncotarget. 2017;8:47841-8.

10. Cerantola $Y$, Valerio $M$, Persson $B$, Jichlinski $P$, Ljungqvist $O$, Hubner $M$, Kassouf W, Muller S, Baldini G, Carli F, et al. Guidelines for perioperative care after radical cystectomy for bladder cancer: Enhanced Recovery After Surgery $\left(\right.$ ERAS $^{\circledR}$ ) society recommendations. Clin Nutr. 2013;32:879-87.
11. Yang R, Tao W, Chen Y-y, Zhang B-h, Tang J-m, Zhong S, Chen X-X. Enhanced recovery after surgery programs versus traditional perioperative care in laparoscopic hepatectomy: a meta-analysis. Int J Surg. 2016:36:274-82.

12. Zhao JH SJ, Gao P, Chen XW, Song YX, Huang XZ, Xu HM, Wang ZN. Fasttrack surgery versus traditional perioperative care in laparoscopic colorectal cancer surgery: a meta-analysis. BMC Cancer. 2014;14:607. doi:10.1186/14712407-14-607.

13. Li P, Fang F, Cai JX, Tang D, Li QG, Wang DR. Fast-track rehabilitation vs conventional care in laparoscopic colorectal resection for colorectal malignancy: a meta-analysis. World J Gastroenterol. 2013;19:9119-26.

14. Wang $D$, Kong $Y$, Zhong B, Zhou $X$, Zhou $Y$. Fast-track surgery improves postoperative recovery in patients with gastric cancer: a randomized comparison with conventional postoperative care. J Gastrointest Surg. 2010; 14:620-7.

15. Li YJ, Huo TT, Xing J, An JZ, Han ZY, Liu XN, Zhao QC. Meta-analysis of efficacy and safety of fast-track surgery in gastrectomy for gastric cancer. World J Surg. 2014;38:3142-51.

16. Ding JSB, Song P, Liu S, Chen H, Feng M, Guan W. The application of Enhanced Recovery After Surgery/fast track surgery in gastrectomy for gastric cancer: a systematic review and meta-analysis. Oncotarget. 2017:8: 75699-711.

17. Kim JW, Kim WS, Cheong J-H, Hyung WJ, Choi S-H, Noh SH. Safety and efficacy of fast-track surgery in laparoscopic distal gastrectomy for gastric cancer: a randomized clinical trial. World J Surg. 2012;36:2879-87.

18. Chen Hu J, Xin Jiang L, Cai L, Tao Zheng H, Yuan Hu S, Bing Chen H, Chang Wu G, Fei Zhang Y, Chuan Lv Z. Preliminary experience of fast-track surgery combined with laparoscopy-assisted radical distal gastrectomy for gastric cancer. J Gastrointest Surg. 2012;16:1830-9.

19. Abdikarim I, Cao XY, Li SZ, Zhao YQ, Taupyk Y, Wang Q. Enhanced recovery after surgery with laparoscopic radical gastrectomy for stomach carcinomas. World J Gastroenterol. 2015;21:13339-44.

20. Moher D, Liberati A, Tetzlaff J, Altman DG, Group P. Preferred reporting items for systematic reviews and meta-analyses: the PRISMA statement. BMJ. 2009;339:b2535.

21. Mortensen K, Nilsson M, Slim K, Schafer M, Mariette C, Braga M, Carli F, Demartines N, Griffin SM, Lassen K. Enhanced recovery after surgery G: consensus guidelines for enhanced recovery after gastrectomy: Enhanced Recovery After Surgery (ERAS $\left.{ }^{\circledR}\right)$ society recommendations. Br J Surg. 2014;101:1209-29.

22. Higgins JP, Altman DG, Gotzsche PC, Juni P, Moher D, Oxman AD, Savovic J, Schulz KF, Weeks L, Sterne JA, et al. The Cochrane Collaboration's tool for assessing risk of bias in randomised trials. BMJ. 2011;343:d5928.

23. Lu J, Huang C-m, Zheng C-h, Li P, Xie J-w, Wang J-bb, Lin J-x, Chen Q-y, Cao L-I, Lin M: Short- and long-term outcomes after laparoscopic versus open total gastrectomy for elderly gastric cancer patients: a propensity score-matched analysis. J Gastrointest Surg 2015, 19:1949-1957.

24. Li YQJ, CaO H. Application of enhanced recovery after surgery for patients with laparoscopic radical gastrectomy. Zhonghua Wei Chang Wai Ke Za Zhi. 2016;19:269-73.

25. Zou ZH, Zhao LY, Mou TY, YF H, Yu J, Liu H, Chen H, JM W, An SL, Li GX Laparoscopic vs open D2 gastrectomy for locally advanced gastric cancer: a meta-analysis. World J Gastroenterol. 2014:20:16750-64.

26. Chen K, Xu XW, Mou YP, Pan Y, Zhou YC, Zhang RC, Wu D. Systematic review and meta-analysis of laparoscopic and open gastrectomy for advanced gastric cancer. World J Surg Oncol. 2013;11:182.

27. Wang JF, Zhang SZ, Zhang NY, ZY W, Feng JY, Ying LP, Zhang JJ. Laparoscopic gastrectomy versus open gastrectomy for elderly patients with gastric cancer: a systematic review and meta-analysis. World J Surg Oncol. 2016;14:90

28. Chen S, Zou Z, Chen F, Huang Z, Li G. A meta-analysis of fast track surgery for patients with gastric cancer undergoing gastrectomy. Ann R Coll Surg Engl. 2015;97:3-10.

29. Bu J, Li N, Huang $X$, He S, Wen J, Wu X. Feasibility of fast-track surgery in elderly patients with gastric cancer. J Gastrointest Surg. 2015;19:1391-8.

30. Feng FJG, Li JP, Li XH, Shi H, Zhao ZW, GS W, Liu XN, Zhao QC. Fast-track surgery could improve postoperative recovery in radical total gastrectomy patients. World J Gastroenterol. 2013;19:3642-8.

31. Petrowsky H, Demartines N, Rousson V, Clavien P-A. Evidence-based value of prophylactic drainage in gastrointestinal surgery. Ann Surg. 2004;240: 1074-85. 
32. JO R. Surgical drainage: an historical perspective. Br J Surg. 1986;73:422-6.

33. Lee J, Choi YY, An JY, Seo SH, Kim DW, Seo YB, Nakagawa M, Li S, Cheong $\mathrm{JH}$, Hyung WJ, Noh SH. Do all patients require prophylactic drainage after gastrectomy for gastric cancer? The experience of a high-volume center. Ann Surg Oncol. 2015;22:3929-37.

34. Conlon KC, Labow D, Leung D, Smith A, Jarnagin W, Coit DG, Merchant N, Brennan MF. Prospective randomized clinical trial of the value of intraperitoneal drainage after pancreatic resection. Ann Surg. 2011;234:487-93.

35. Slim K, Fingerhut A. Laparoscopy or fast-track surgery, or both? Surg Endosc. 2009:23:465-6

36. Veenhof AA, Vlug MS, van der Pas MH, Sietses $C$, van der Peet $D L$, de Lange-de Klerk ES, Bonjer HJ, Bemelman WA, Cuesta MA. Surgical stress response and postoperative immune function after laparoscopy or open surgery with fast track or standard perioperative care: a randomized trial. Ann Surg. 2012;255:216-21.

37. Kawamura H, Yokota R, Homma S, Kondo Y. Comparison of respiratory function recovery in the early phase after laparoscopy-assisted gastrectomy and open gastrectomy. Surg Endosc. 2010;24:2739-42.

38. Feng J, Li K, Li L, Wang X, Huang M, Yang J, Hu Y. The effects of fast-track surgery on inflammation and immunity in patients undergoing colorectal surgery. Int J Color Dis. 2016;31:1675-82.

39. Chen L, Sun L, Lang Y, Wu J, Yao L, Ning J, Zhang J, Xu S.Fast-track surgery improves postoperative clinical recovery and cellular and humoral immunity after esophagectomy for esophageal cancer. BMC Cancer. 2016;16:449. doi:10.1186/s12885-016-2506-8

40. Liu G, Jian F, Wang X, Chen L. Fast-track surgery protocol in elderly patients undergoing laparoscopic radical gastrectomy for gastric cancer: a randomized controlled trial. Onco Targets Ther. 2016;9:3345-51.

41. Senkal M, Zumtobel V, Bauer KH, Marpe B, Wolfram G, Frei A, Eickhoff U, Kemen M. Outcome and cost-effectiveness of perioperative enteral immunonutrition in patients undergoing elective upper gastrointestinal tract surgery: a prospective randomized study. Arch Surg. 1999;134:1309-16.

42. Yan X, Zhou FX, Lan T, Xu H, Yang XX, Xie CH, Dai J, ZM F, Gao Y, Chen LL. Optimal postoperative nutrition support for patients with gastrointestinal malignancy: a systematic review and meta-analysis. Clin Nutr. 2017;36:710-21.

43. Shu XL, Kang K, LJ G, Zhang YS. Effect of early enteral nutrition on patients with digestive tract surgery: a meta-analysis of randomized controlled trials. Exp Ther Med. 2016;12:2136-44.

44. Lewis SJ, Andersen HK, Thomas S. Early enteral nutrition within $24 \mathrm{~h}$ of intestinal surgery versus later commencement of feeding: a systematic review and meta-analysis. J Gastrointest Surg. 2009;13:569-75.

45. Nikniaz Z, Somi MH, Nagashi S, Nikniaz L. Impact of early enteral nutrition on nutritional and immunological outcomes of gastric cancer patients undergoing gastrostomy: a systematic review and meta-analysis. Nutr Cancer. 2017;69:693-701.

\section{Submit your next manuscript to BioMed Central and we will help you at every step:}

- We accept pre-submission inquiries

- Our selector tool helps you to find the most relevant journal

- We provide round the clock customer support

- Convenient online submission

- Thorough peer review

- Inclusion in PubMed and all major indexing services

- Maximum visibility for your research

Submit your manuscript at www.biomedcentral.com/submit

) Biomed Central 